\title{
Efficacy and safety of lenvatinib in an elderly patient with metastatic papillary thyroid carcinoma and cardiological comorbidity: a case report
}

\author{
Agnese Latorre*,1, Agnese Maria Fioretti ${ }^{2}$, Francesco Giotta ${ }^{1}$ \& Vito Lorusso ${ }^{1}$ \\ ${ }^{1}$ UO Oncologia Medica, Istituto Tumori "G Paolo II" Bari, Bari, Italy \\ ${ }^{2}$ UO Dipartimentale di Cardiologia, Istituto Tumori "G Paolo II" Bari, Bari, Italy \\ *Author for correspondence: Tel.: +39080 555 5427; agnelato@libero.it
}

Lenvatinib significantly prolonged progression-free survival versus placebo in patients with radio-iodine refractory differentiated thyroid carcinoma. However, the primary adverse effects of any grade that occurred in $>40 \%$ of patients in the lenvatinib group of the Phase III SELECT trial was hypertension (67.8\%). Therefore, this drug should be used with caution in patients with cardiological morbidity. Here, we describe the case of a 73-year-old man with hypertension, obesity and chronic atrial fibrillation, who received lenvatinib for 6 months in the absence of cardiological symptoms.

First draft submitted: 27 February 2019; Accepted for publication: 16 July 2019; Published online: 8 August 2019

Keywords: cardiological comorbidity • lenvatinib $\bullet$ papillary thyroid cancer $\bullet$ tyrosine kinase inhibitor

Outcomes of differentiated thyroid carcinoma (DTC) treatment are usually favorable. Surgery is the standard treatment for locally advanced tumors, with radioactive iodine $\left({ }^{131} \mathrm{I}\right)$ therapy in selected cases $[1,2]$. Conversely, recurrent or metastatic radioactive iodine (RAI) refractory disease has a poor prognosis, with a 10-year survival rate as low as $15-20 \%[1,2]$. Two tyrosine kinase inhibitors (TKIs), sorafenib and lenvatinib, are now approved in the USA and Europe for the treatment of RAI refractory DTC. Both agents were approved based on progression-free survival in previous placebo-controlled, Phase III trials [3,4]. Both of these multiple targeted TKIs have various adverse events (AEs) leading to dose interruption and reduction, such as hypertension, hand-foot syndrome, eruption, proteinuria, diarrhea, fatigue and hepatic dysfunction [5]. Hand-foot syndrome is the main event after sorafenib treatment [6] and hypertension after lenvatinib [7].

Atrial fibrillation (AF) is commonly diagnosed in the setting of active cancer. Many pathophysiological disorders may arise during cancer treatment, leading to arrhythmias, including mass effect of tumors, dehydration, electrolyte disorders and pain with subsequently increased sympathetic tone [8].

Pre-existing AF was found in 2.4\% of 24,000 patients with newly diagnosed malignancy, at diagnosis, and newonset $\mathrm{AF}$ after their initial cancer diagnosis was reported in $1.8 \%$ of patients [9]. AF in cancer patients was associated with higher rates of hypertension, myocardial infarction and heart failure compared with cancer patients without AF. A twofold increased risk of thromboembolism and a sixfold increased risk of heart failure were associated with new-onset $\mathrm{AF}$ after the diagnosis of malignancy in comparison with patients with $\mathrm{AF}$ at the time of cancer diagnosis. This finding suggests that strict attention should be paid to the occurrence of AF during cancer treatment. The number of cancer survivors in the USA is expected to be about 19 million in 2024 [10], and it is thought that this event is mainly due to the use of new effective and targeted cancer therapies. Among this high number of survivors, many patients will experience AF during treatment or follow-up; so, management of this condition should be well defined.

In this report, we experienced a locally advanced and metastatic DTC patient who had hypertension, obesity and permanent $\mathrm{AF}$, underwent craniotomy with exeresis of the left temporo-parietal brain neoformation compatible with cerebral localization of papillary neoplasm of thyroid origin, with extended and rapid growth of metastatic 


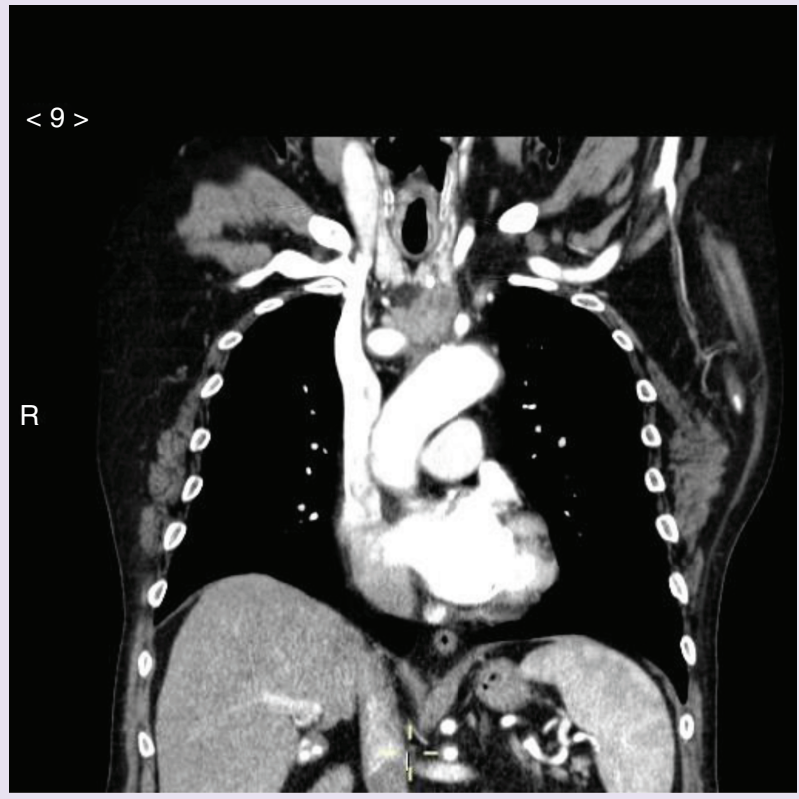

Figure 1. CT scan: increased isthmus of thyroid volume, irregular for hypodense nodular foci and calcifications in the left paramedian, thickening of the anterior mediastinal tissue up to the retrosternal planes.

tumors after the surgery and for whom lenvatinib was proved to be remarkably effective in the absence of more cardiological complications.

\section{Case presentation}

Here, we describe the case of a 73-year-old man, who was a former strong smoker, and affected by hypertension, obesity and chronic AF. In May 2017, the patient demonstrated headache, language disorders and upper right limb hyposthenia. Brain CT scan and MRI found cerebral neoformation with massive perilesional edema in the left parietal site. The patient underwent craniotomy with exeresis of left temporo-parietal brain neoformation.

The pathological report showed cerebral localization of papillary neoplasm of thyroid origin, CK pool (++-), EMA (+++), vimentine (+-) positive, GFAP and CD10-negative, Ki-67 20\%. Staging with total-body CT scan showed pulmonary metastases and colliquated adenopathies in the mediastinum and Barety loggia. Bronchoscopy with brushing and bronchial lavage resulted negative for tumor lesions. A thyroid ultrasound scan revealed chronic thyropathy with latero-cervical inflammatory lymphadenopathies. The postoperative course was regular and complication free.

In August 2017, the patient was hospitalized for dysphagia and dyspnea, and the CT scan showed dense and irregular tissue with a diameter of $3 \times 4 \mathrm{~cm}$ in the anterosuperior mediastinal site associated with Barety loggia, right ileum and prevascular lymphadenopathy. The patient was discharged with a diagnosis of metastatic papillary carcinoma of thyroid origin (T4N1bM1), stage IV according to the seventh edition of the Union for International Cancer Control Tumor, Node, Metastasis (TNM) classification of malignant tumors, without indication to surgery or radiotherapy due to the presence of brain metastases.

The patient arrived under our observation in October 2017. A new CT scan was performed and showed outcomes of left temporoparietal craniotomy. Increased isthmus of thyroid volume $(34 \times 37 \mathrm{~mm})$, irregular for hypodense nodular foci of a few millimeters and calcifications in the left paramedian, thickening of the anterior mediastinal tissue up to the retrosternal planes, engagement in the thoracic strait in retrovascular site, compression of the anterior wall of the trachea. Voluminous right paratracheal adenopathy $(34 \times 22 \mathrm{~mm})$ and other right and prevascularilo-lobar adenopathies. Multiple nodular lesions randomly distributed in both lungs larger than $10.4 \mathrm{~mm}$ at LID (Figures 1-3).

His past medical history included hypertension, dyslipidemia and permanent AF, all controlled using valsartanhydrochlorothiazide, amlodipine and fondaparinux. Moreover, he took dexamethasone and levetiracetam for the 


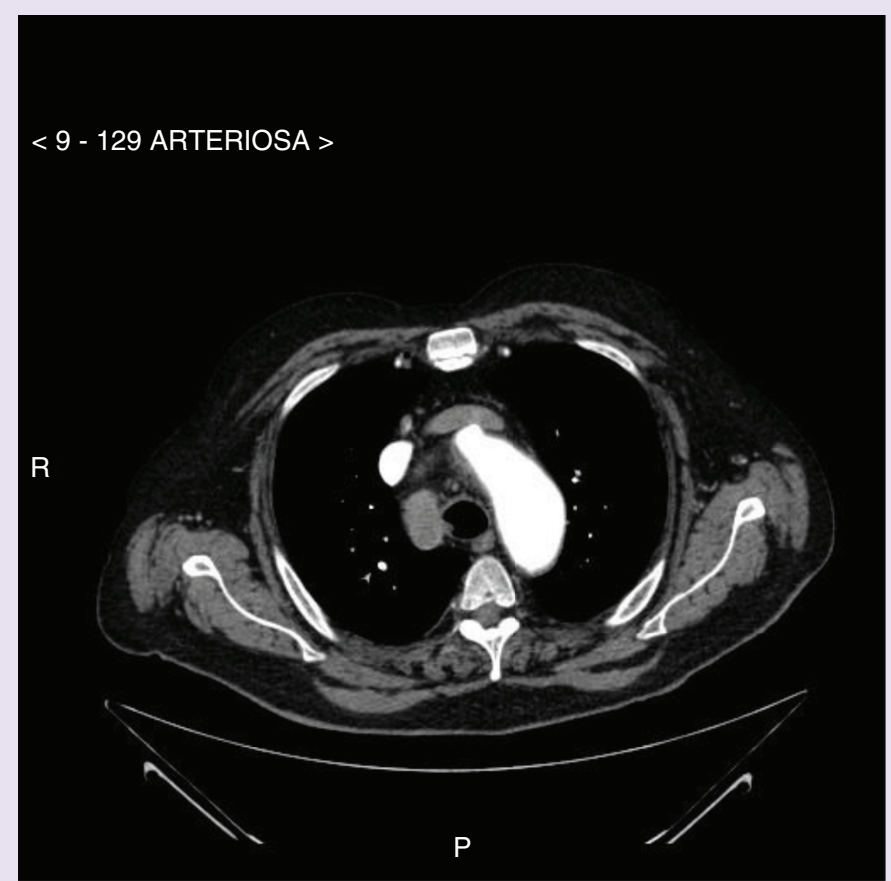

Figure 2. CT scan: voluminous right paratracheal adenopathy $(34 \times 22 \mathrm{~mm})$ and other right and prevascularilo-lobar adenopathies.

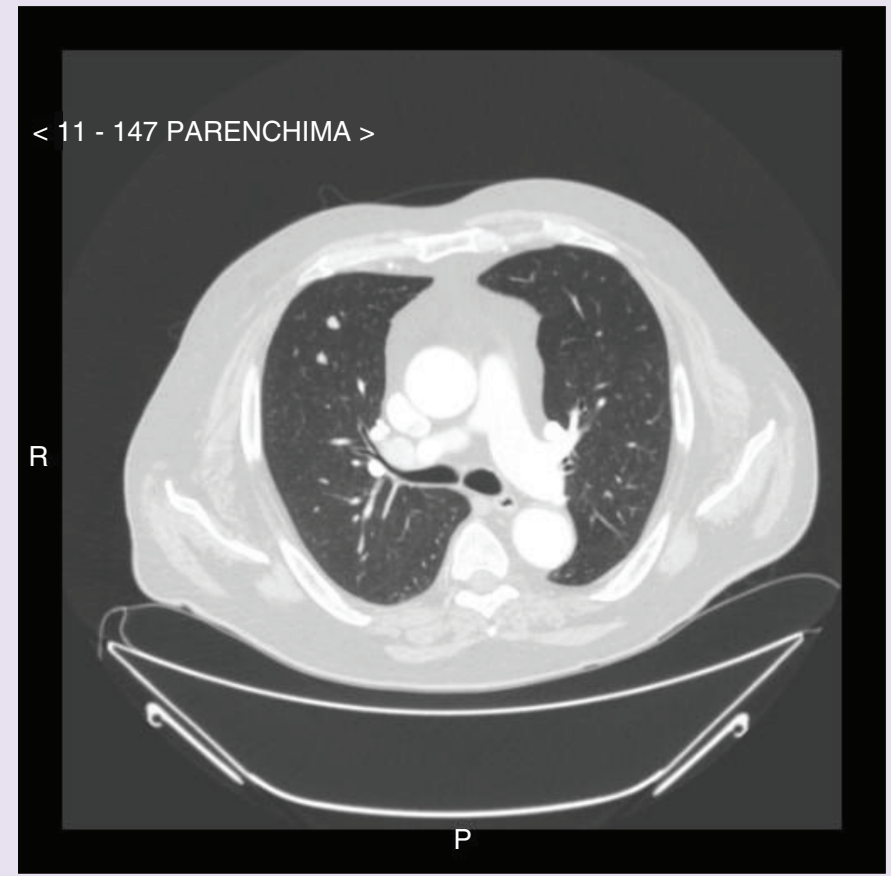

Figure 3. CT scan: multiple nodular lesions randomly distributed in both lungs. 
control of cerebral surgery outcomes. Surgery was contraindicated, in the opinion of thoracic surgeons, because of the poor prognosis associated with encephalic, cervical, mediastinal and diffuse pulmonary metastases, the large size of the mass and the poor performance status. In this patient, multimodal therapies, ${ }^{131} \mathrm{I}$, external-beam radiation therapy and taxane-based chemotherapy were not indicated because of the stage, presence of pulmonary and brain metastases, age and comorbidity. As the US FDA approval of lenvatinib supports its use as a monotherapy in metastatic, progressive or RAI refractory DTC, lenvatinib was initiated as a TKI in November 2017. However, because of chronic AF, after cardiological consultation, the patient started lenvatinib at reduced doses of $10 \mathrm{mg} / \mathrm{die}$ with a weekly increase of $4 \mathrm{mg}$ and close electrocardiographic follow-up and daily control of blood pressure.

After 1 week of taking the $18 \mathrm{mg} /$ day dose, the patient showed a marked subjective improvement with reduction of cervical mass, improvement in dysphagia, dyspnea and pain, but appearance of grade 3 mucositis and hypercholesterolemia (total cholesterol $329 \mathrm{mg} / \mathrm{dl}$ ) on the ECG showed AF at $74 \mathrm{bpm}$ with left axial deviation, left ventricular hypertrophy with systolic overload and QTc interval within the limits and echo color Doppler uniform remarkable wall hypertrophy with left ventricular ejection fraction (LVEF) of $45-50 \%$ and aortic ectasia. Due to mucositis and asthenia, the dose of lenvatinib was reduced to $14 \mathrm{mg} /$ day and continued at the same dose with the addition of simvastatin. With this therapy, the blood pressure values have always remained within the limits. The patient continued therapy with improvement of mucositis (grade 1), asthenia and serum cholesterol values, obtaining a further objective reduction of the thyroid mass. In March 2018, the patient was presented to us with worsening of mucositis and asthenia (grade 2), appearance of abdominal petechiae, depressions in mood, increase in creatinine value for which the required CT scan had been postponed. The scheduled CT scan was not performed because the patient referred to a different hospital.

The patient did not show up at the next scheduled monthly appointment. Traced by phone, he reported that had stopped the therapy because worsening of mucositis and asthenia and had gone to another hospital. The patient's clinical conditions had significantly improved after lenvatinib therapy. Both pain and dysphagia were reduced and the patient could eat, so that nutrition was better. On the basis of the clinical and instrumental reassessment carried out in April 2018 at the Pisa hospital, total thyroidectomy with emptying of the central compartment was scheduled, in order to allow the patient to receive radiometabolic therapy with high doses of ${ }^{131} \mathrm{I}$.

On 5 November 2018, the patient underwent total thyroidectomy surgery and mediastinal lesion biopsy with evidence of solid papillary carcinoma with widespread multifocal 'hobnail aspects' infiltrating the perithyroid tissues with vascular embolization and mediastinal localization.

The postoperative course has been regular and without complications. The patient is awaiting to undergo radiation treatment, aimed at obtaining euthyroidism after stimulation with recombinant human TSH (rhTSH).

\section{Discussion \& conclusion}

The standard treatment of locally advanced DTC is surgery achieving R0 (no residual cancer) [11,12]. In this case, there was no indication of surgery or radiotherapy due to the presence of pulmonary and brain metastases, with a large tumor size, and a poor general health. RAI therapy in the first line is indicated after thyroidectomy. Thyroidectomy was excluded in our patient due to the presence of brain metastases, which ruled out the choice of RAI. The patient was treated with lenvatinib and experienced a subjective improvement; unfortunately, no instrumental data are available to prove the disease state after lenvatinib therapy, because the patient did not undergo the requested CT scan and was lost to follow-up.

Median progression-free survival of patients treated with lenvatinib was significantly higher compared with subjects who had received a placebo, in a randomized trial [4]. Lenvatinib has also a RET inhibiting action resulting in a reduced tumor cell proliferation, and anti-oncogenic effect. In addition, it blocks FGFR, modulating the tumor microenvironment [13,14].

Lenvatinib has been associated with numerous serious vascular adverse effects, including severe hypertension, thromboembolic events and hemorrhagic strokes [4]. Hypertension is among the most common AEs related to lenvatinib reported in Phase II and Phase III studies. Among DTC patients treated with lenvatinib under the SELECT trial, $68 \%$ of the subjects developed treatment-related hypertension. Other frequent AEs in the SELECT study were diarrhea (59.4\%), fatigue or asthenia (59.0\%), decreased appetite (50.2\%), decreased weight (46.4\%), nausea (41.0\%), stomatitis (35.6\%), palmar-plantar erythrodysesthesia syndrome (31.8\%) and proteinuria (31.0\%) [15]. These common AEs associated with lenvatinib are typical of the AEs reported more generally with TKIs in patients with thyroid cancer $[15,16]$. The dose was reduced in $78.5 \%$ of patients and $14.2 \%$ discontinued because of poor drug tolerance [17]. 
Lenvatinib had no clinically relevant effect on the QTc interval in 52 healthy volunteers who randomly received single doses of lenvatinib $32 \mathrm{mg}$. Concentration-effect modeling supports the lack of QTc prolongation at high plasma concentrations [18].

In clinical studies with lenvatinib, cardiac dysfunction was reported in $7 \%$ of lenvatinib-treated patients and $2 \%$ of placebo-treated patients. A decreased ejection fraction was found in most patients who underwent an echocardiography. The ejection fraction was reduced by $>20 \%$ in $2 \%$ of patients treated with lenvatinib, and was not reduced in any patients in the group treated with placebo. A total of $73 \%$ of patients treated with lenvatinib, and $16 \%$ of those receiving placebo had blood hypertension. In studies with lenvatinib, arterial thromboembolic events were reported in 5\% of lenvatinib-treated patients and $2 \%$ of patients in the placebo group. In total, $3 \%$ of patients receiving lenvatinib, and $1 \%$ of those treated with placebo, had grade $\geq 3$ AEs [19].

A cross-sectional study on a limited sample found that an initial low dose of lenvatinib was not associated with a better tolerability [20].

At baseline, 16-36\% of patients treated for a cancer have an arrhythmia. In addition, cancer therapies, imbalance of electrolytes and concomitant drugs, such as antiemetics, cariological drugs, antibiotics and psychiatric medications, can induce QT prolongation or facilitate serious arrhythmias (e.g., torsade de pointes). All risk factors, including QT interval duration, should be checked before, during and after cancer treatment. Arsenic trioxide has the highest risk for QT prolongation, but also TKIs, and particularly vandetanib are associated with an increased risk (vandetanib immediately after arsenic trioxide) [21].

$\mathrm{AF}$ is commonly diagnosed in the setting of active cancer and $2.4 \%$ of patients with newly diagnosed malignancy had pre-existing $\mathrm{AF}$ at the time of their cancer diagnosis. AF was also diagnosed in $1.8 \%$ of patients after the diagnosis of cancer [9]. Cancer patients have a higher prevalence of AF than the general population (approximately 1\%) [22]. This likely involves greater incidence of hypertension, myocardial infarction and heart failure in patients with cancer and AF compared with those patients who did not develop AF. Commonly used cancer treatments may also contribute to the development of AF [23]. In particular, targeted therapies such as the TKI, are associated with a risk for AF and for drug-drug interactions. In addition, the management of AF may be difficult because of possible drug-drug interactions between targeted cancer therapies and antiarrhythmic drugs (AADs). Published evidence on concomitant administration of oral anticoagulants and targeted therapies for cancer is scant. Nevertheless, drug interactions between cardiovascular drugs and targeted therapies, whose number is growing, must be studied as co-medications are rarely encountered in the clinical practice.

Each one of the new cancer therapies is directed toward several targets, and has a different kinome binding profile with a different pharmacokinetic/AE profile. Concomitant administration of targeted cancer therapies and AADs may induce high drug concentrations. The coadministration of AADs with some targeted therapies, although associated with a risk for bradycardia and QT prolongation can be used with careful electrocardiographic monitoring and/or dose reduction, but some associations are contraindicated, and this may represent an obstacle to the treatment of cancers in some conditions. Moreover, the management AF in cancer patients must be careful, as the use of anticoagulants is not recommended. Finally, it can be mentioned that use of targeted therapies can be long-term nowadays, as survival to several cancers has increased and tolerability of novel products is good.

\section{Summary points}

- Hypertension is a frequent adverse event associated with lenvatinib. It was the most common adverse event in SELECT study ( $67.8 \%$ of subjects).

- Lenvatinib should be used with caution in patients with cardiological morbidity.

- A 73-year-old man with hypertension, obesity and chronic atrial fibrillation received lenvatinib for 6 months without occurrence of cardiological symptoms.

Financial \& competing interests disclosure

The authors have no relevant affiliations or financial involvement with any organization or entity with a financial interest in or financial conflict with the subject matter or materials discussed in the manuscript. This includes employment, consultancies, honoraria, stock ownership or options, expert testimony, grants or patents received or pending, or royalties.

Editorial assistance was provided by A Shah and L Giacomelli (Polistudium srl, Milan, Italy), on behalf of Content Ed Net. This assistance was supported by Eisai. 
Informed consent

Written informed consent was obtained from the patient for publication of this case report and the accompanying images.

\section{Open access}

This work is licensed under the Attribution-NonCommercial-NoDerivatives 4.0 Unported License. To view a copy of this license, visit http://creativecommons.org/licenses/by-nc-nd/4.0/

\section{References}

Papers of special note have been highlighted as: • of interest; $\bullet \bullet$ of considerable interest

1. Gruber JJ, Colevas AD. Differentiated thyroid cancer: focus on emerging treatments for radioactive iodine-refractory patients. Oncologist 20(2), 113-126 (2015).

2. Schmidt A, Iglesias L, Klain M, Pitoia F, Schlumberger MJ. Radioactive iodine refractory differentiated thyroid cancer: an uncommon but challenging situation. Arch. Endocrinol. Metab. 61(1), 81-89 (2017).

3. Brose MS, Nutting CM, Jarzab B et al. Sorafenib in radioactive iodine-refractory, locally advanced or metastatic differentiated thyroid cancer: a randomised, double-blind, Phase III trial. Lancet 384, 319-328 (2014).

4. Schlumberger M, Tahara M, Wirth LJ et al. Lenvatinib versus placebo in radioiodine-refractory thyroid cancer. N. Engl. J. Med. 372, 621-630 (2015).

-• Pivotal study for lenvatinib efficacy and tolerability

5. Brose MS, Smit J, Lin CC et al. Timing of multikinase inhibitor initiation in differentiated thyroid cancer. Endocr. Relat. Cancer 24(5), 237-242 (2017).

6. Pitoia F, Jerkovich F. Selective use of sorafenib in the treatment of thyroid cancer. Drug Des. Devel. Ther. 10, 1119-1131 (2016).

7. Sueta D, Suyama K, Sueta A et al. Lenvatinib, an oral multi-kinases inhibitor, -associated hypertension: potential role of vascular endothelial dysfunction. Atherosclerosis 260, 116-120 (2017).

8. Bayraktar UD, Dufresne A, Bayraktar S, Purcell RR, Ajah OI. Esophageal cancer presenting with atrial fibrillation: a case report. J. Med. Case Rep. 2, 292 (2008).

9. Hu YF, Liu CJ, Chang PM et al. Incident thromboembolism and heart failure associated with new-onset atrial fibrillation in cancer patients. Int. J. Cardiol. 165, 355-357 (2013).

10. De Santis CE, Lin CC, Mariotto AB et al. Cancer treatment and survivorship statistics, 2014. CA Cancer J. Clin. 64, 252-271 (2014).

11. Wang LY, Nixon IJ, Patel SG et al. Operative management of locally advanced differentiated thyroid cancer. Surgery 160(3), 738-746 (2016).

12. Wang LY, Ghossein R, Palmer FL et al. Microscopic positive margins in differentiated thyroid cancer is not an independent predictor of local failure. Thyroid 25(9), 993-998 (2015).

13. Tohyama O, Matsui J, Kodama K et al. Antitumor activity of lenvatinib (e7080): an angiogenesis inhibitor that targets multiple receptor tyrosine kinases in preclinical human thyroid cancer models. J. Thyroid Res. 2014, 638747 (2014).

14. Stjepanovic N, Capdevila J. Multikinase inhibitors in the treatment of thyroid cancer: specific role of lenvatinib. Biologics 8, 129-139 (2014).

15. Cabanillas ME, Takahashi S. Managing the adverse events associated with lenvatinib therapy in radioiodine-refractory differentiated thyroid cancer. Semin. Oncol. 46(1), 57-64 (2019).

- Relevant for practice.

16. Pitoia A, Schmidt A, Bueno F, Abelleira E, Jerkovich F. Rare complications of multikinase inhibitor treatment. Arch. Encocr. Melab. 62(6), 636-640 (2018).

17. Krajewska J, Jarzab B. Novel therapies for thyroid cancer. Expert Opin. Pharmacother. 15(18), 2641-2652 (2014).

18. Shumaker RC, Zhou M, Ren M et al. Effect of lenvatinib on the QT-c interval: results from a through QT study in healthy volunteers. Cancer Chemother. Pharmacol. 73, 1109-1117 (2014).

19. Shah RR, Morganroth J. Update on cardiovascular safety of tyrosine kinase inhibitors: with a special focus on QT interval, left ventricular dysfunction and overall risk/benefit. Drug Saf. 38, 693-710 (2015).

20. Yamazaki $\mathrm{H}$, Iwasaki $\mathrm{H}$, Takasaki $\mathrm{H}$ et al. Efficacy and tolerability of initial low-dose lenvatinib to treat differentiated thyroid cancer. Medicine (Baltimore) 98(10) , e14774 (2019).

21. Zamorano JL, Lancellotti P, Rodriguez Muñoz D et al. ESC Scientific Document Group. 2016 ESC Position Paper on cancer treatments and cardiovascular toxicity developed under the auspices of the ESC Committee for Practice Guidelines: the task force for cancer treatments and cardiovascular toxicity of the European Society of Cardiology (ESC). Eur. Heart J. 37(36), 2768-2801 (2016).

-. Reviews the different steps in cardiovascular monitoring and decision-making before, during and after anticancer treatment with potential cardiovascular side effects. 
22. Go AS, Hylek EM, Phillips KA et al. Prevalence of diagnosed atrial fibrillation in adults: national implications for rhythm management and stroke prevention: the AnTicoagulation and risk factors in atrial fibrillation (ATRIA) study. JAMA 285, 2370-2375 (2001).

23. Guglin M, Aljayeh M, Saiyad S, Ali R, Curtis AB. Introducing a new entity: chemotherapy-induced arrhythmia. Europace 11, 1579-1586 (2009). 